\title{
An inexpensive sound-activated switch for experiments measuring vocal-response times
}

\author{
KEVIN J. HAWLEY and EUGENE J. IZATT \\ University of Utah, Salt Lake City, Utah
}

\begin{abstract}
An inexpensive sound-activated switch suitable for experiments measuring vocal-response times is described. This switch is comparable to many commercially produced switches in terms of its control over input sensitivity and response delay times, but it can be assembled for a fraction of the cost. Additionally, the present switch provides many features not found on standard commercial models such as a built-in speaker for monitoring the input signal and output jacks to facilitate simultaneous recording of this signal. Reliability estimates suggest that performance for this switch is comparable to that of a Scientific Prototype Model 761-G when both units are equated for frequency sensitivity and response delay time. In all, this switch offers a reliable and practical alternative to commercially produced devices for experiments in which the onset of an auditory signal must be accurately detected.
\end{abstract}

Over the past decade, increasing numbers of studies have utilized vocal-response times as a primary index of cognitive performance. The specific applications range from investigations of priming and word recognition (e.g., Dark, 1988; Johnston, Hawley, \& Elliott, 1991) to those of picture identification (Glaser \& Dungelhoff, 1984), reading and lexical access (Brown, Neblett, Jones, \& Mitchell, 1991; Levelt et al., 1991), and Stroop interference (Glaser \& Glaser, 1982). As a result, several scientific instrumentation companies (e.g., Gerbrands, Lafayette, Scientific Prototype) now produce soundactivated switching devices that can be used to detect vocal-response onsets. Unfortunately, although these commercially available devices provide reliable estimates of vocal-response times, they are often very expensive (e.g., $\$ 730$ for the Gerbrands Model G1341-T), and, other than controls for sensitivity to the incoming signal and delay times for the generated response, they provide little in the way of additional features. ${ }^{1}$ The present paper describes a compact, portable, and inexpensive soundactivated switch suitable for studies investigating vocal-

The preparation of this manuscript was supported in part by a University of Utah Graduate Research Fellowship to the first author and by Air Force Office of Scientific Research Grant 89-0275 to William Johnston at the University of Utah. We would like to thank William Johnston and Jim Farnham for their helpful comments on an earlier draft of this paper. We also wish to acknowledge Radio Shack for permission to publish the schematic in Figure 3. Correspondence concerning this paper should be addressed to K. J. Hawley, Department of Psychology, University of Utah, Salt Lake City, UT 84112. Requests for detailed technical information should be addressed to $E$. J. Izatt, Department of Psychology, University of Utah, Salt Lake City, UT 84112. response times or other tasks in which the onset of an auditory signal must be accurately detected.

\section{SWITCH CHARACTERISTICS}

Typically, sound-activated switches are used to mediate the signals between an auditory input device (e.g., a microphone) and a recording device (e.g., a microcomputer). The switch suppresses all signal activity from the input device to the recording device until the level of the input signal exceeds some discrete threshold. The suppression of all low-level input signals allows the recording device to ignore irrelevant signals generated from background noise and to record only suprathreshold signals such as those produced by a deliberate vocalresponse. When a sufficiently intense input signal is detected, the switch indicates the detection of this signal to the recording device. In general, there are two ways in which this indication can be made. The first method involves the generation of a response pulse by the switch along the input line to the recording device. The second method involves the closing of a relay by the switch, which results in a complete circuit along the input line with the recording device. Both methods result in a flow of current to the recording device's input port. When the recording device is a microcomputer, the flow of current along this circuit usually serves to set some bit(s) in the input port's data buffer and signals the CPU to take some appropriate action, such as logging the contents of the screen or recording the time at which the incoming signal was detected. The primary difference between these two methods is that while the generation of current along 
the input line is actively produced by the switch in the former case, it must be generated by the recording device (or some other external source) in the latter. Although most commercial units use the first of these two methods, the second method is somewhat more flexible in that it allows the experimenter to decide what type of signal to send and receive.

The most crucial features on any sound-activated switch are the range of auditory frequencies to which the switch will respond and the characteristics of the response signal that is generated. ${ }^{2}$ Flexibility of control over these parameters varies widely among commercial models. For example, although the Scientific Prototype Model 761-G allows for input sensitivity to be tailored to particular frequencies in the low, middle, and high bands of the auditory spectrum as well as provides control over the attack and decay rates of the generated signal, the Gerbrands Model G1341-T only allows for a global sensitivity level to be specified, and only the duration of the response pulse can be manipulated. For the purposes of detecting discrete (i.e., single utterance) vocal responses the differences in level of control provided by these different models are not as great as they may at first appear. ${ }^{3} \mathrm{Be}$ cause human vocal responses occupy a relatively narrow band of the auditory spectrum, it is usually unnecessary for the purposes of simple signal detection to tailor the input sensitivity to several subsets of this frequency range. Moreover, because digital recording devices such as microcomputers typically detect only the presence or absence of a signal, the overall intensity or rate of change in a response signal is ignored as long as the absolute level of the response exceeds some minimum threshold (e.g., $12 \mathrm{~V}$ on a standard RS-232 serial card). For this reason, the performance of switches that provide fewer controls over input sensitivity and response generation does not differ significantly from that of more selective switches when the former are used for simple signal detection in vocal-response-time experiments.

The sound-activated switch described below is similar in terms of its control over input sensitivity and response generation to the Gerbrands Model G1341-T. However, in addition to providing several features not found on the Gerbrands switch, the present switch is smaller, more light-weight, and significantly less expensive than its Gerbrands counterpart. We will first describe the overall characteristics and features present on the switch before detailing the components used in its construction.

\section{SWITCH DESCRIPTION}

\section{Overall Features and Characteristics}

A diagram of our switch is given in Figure 1. In general, the unit is $13 \mathrm{~cm}$ wide, $7 \mathrm{~cm}$ tall, and $11 \mathrm{~cm}$ deep with a weight of approximately $432 \mathrm{~g}(15.2 \mathrm{oz})$. It utilizes a 9-V dc power source generated by an external adaptor and provides controls for adjusting both the unit's sensitivity to an input signal (i.e., the minimum input signal level required to produce a response) and the duration of the generated response. All of the switch's I/O ports are either RCA or phone standard input jacks. In addition to providing an output jack for indicating signal detection to a recording device, our switch also provides output jacks for routing the incoming audio signal to both an external speaker and an external recording device (e.g., a tape recorder). The incoming signal can also be monitored by the experimenter via an internal speaker or through a headset connected to a special jack on the back of the switch's casing. The input signal is assumed to be an analog audio signal originating from either a microphone or an external amplifier. All input and output signals are compatible with standard low-impedance devices (i.e., microphones, headphones, etc., with impedances in the 50-600 $\Omega$ range). The switch itself has an input impedance of approximately $5 \mathrm{k} \Omega$ with a total distortion of less than $2 \%$. It has an input sensitivity threshold of $1 \mathrm{mV}$ and will respond to frequencies between $100 \mathrm{~Hz}$ and $10 \mathrm{kHz}$. Although some first formant information in human speech does extend down to $50 \mathrm{~Hz}$, the majority of frequencies typically fall between $100 \mathrm{~Hz}$ and $5 \mathrm{kHz}$ (Borden \& Harris, 1980). As a result, the smaller frequency response range for our switch relative to some commercial models (e.g., $30 \mathrm{~Hz}$ to $40 \mathrm{kHz}$ for the Gerbrands Model G1341-T) does not dramatically lessen its ability to detect human vocal responses.

\section{Components}

A block diagram of the switch construction and logic is given in Figure 2. A schematic diagram along with a parts list and cost breakdown is provided in Figure 3. In
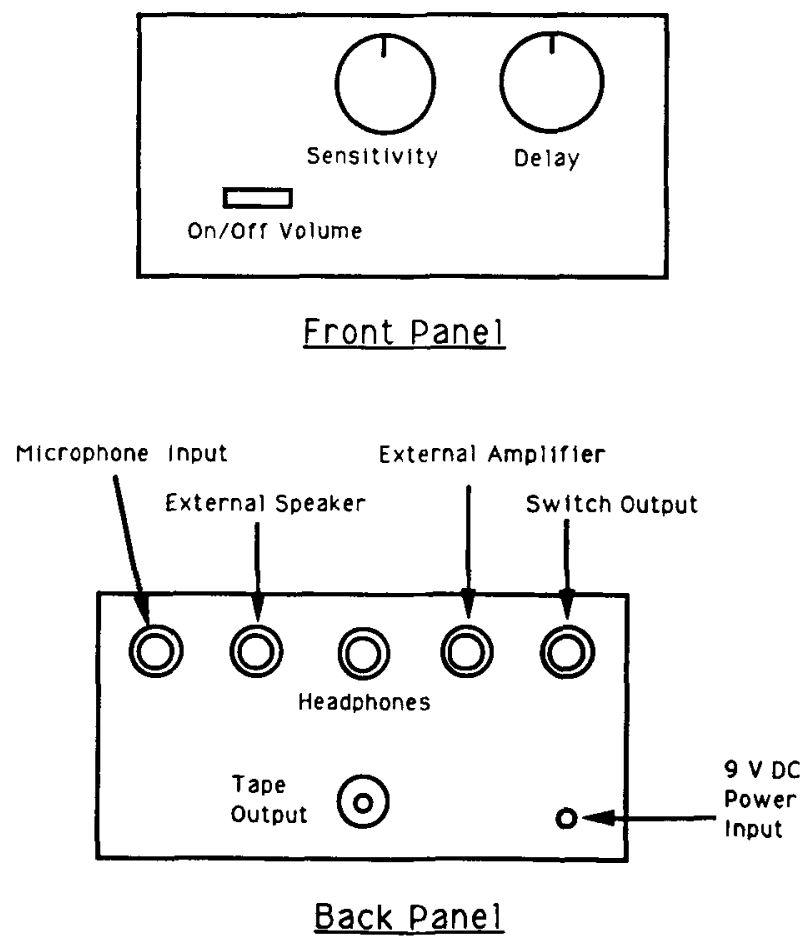

Figure 1. A general diagram depicting the front and back panels of our switch. 


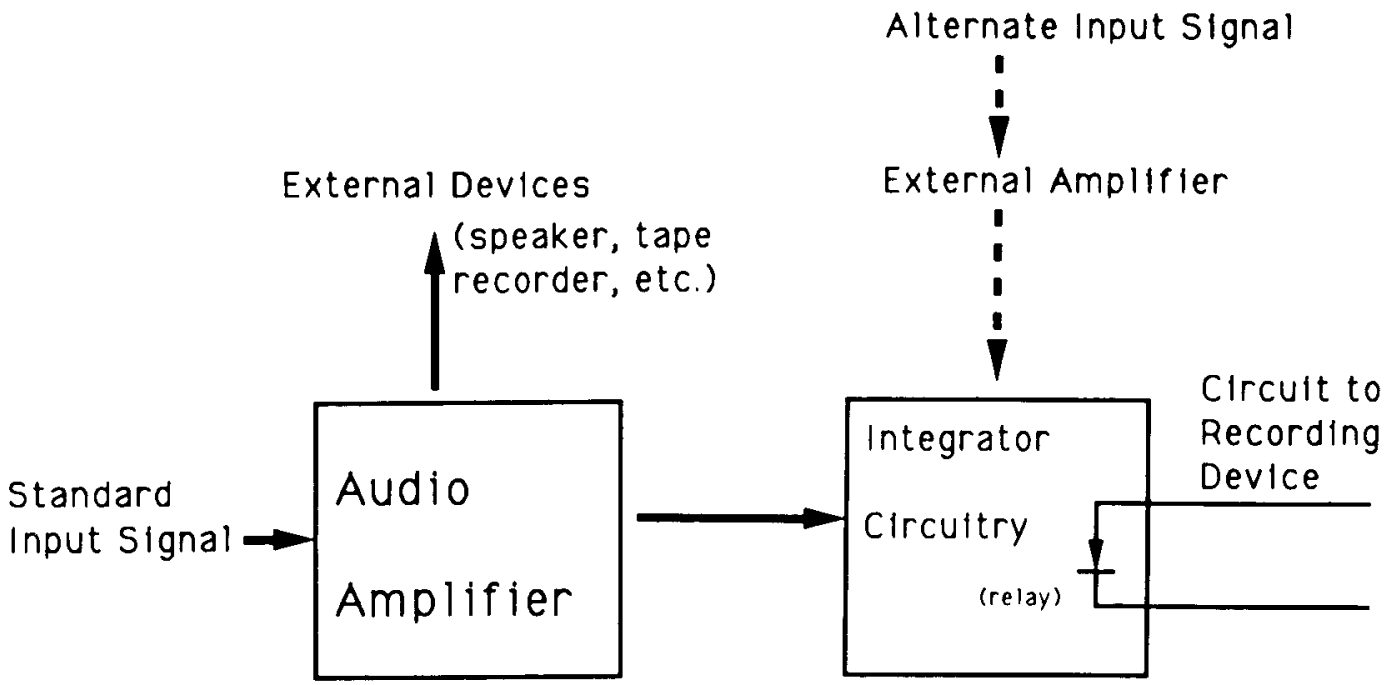

Figure 2. A block diagram of the circuit logic employed in our switch. Audio input to the integrator circuitry is obtained from either the built-in amplifier or an external amplifier. Detection of a suprathreshold signal closes a relay and completes a circuit along the output line with a recording device.

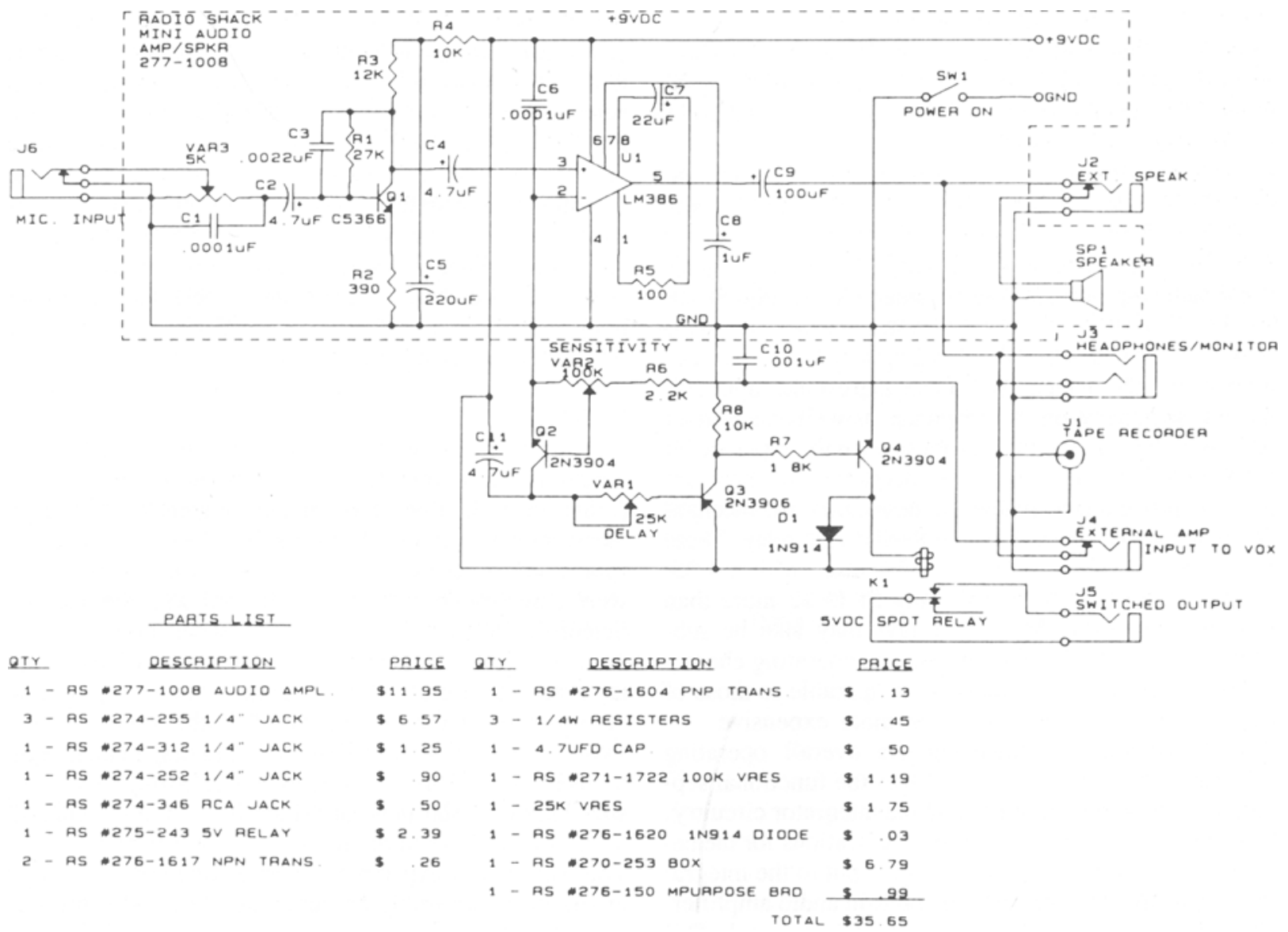

Figure 3. A schematic diagram and cost breakdown for each of the switch's components. All components inside the dotted line are contained in the Radio Shack audio amplifier. Components outside the dotted line comprise the integrator circuitry and $\mathbf{I} / \mathrm{O}$ jacks. 
general, the unit consists of two main components. The first of these is a small audio amplifier (Radio Shack No. 277-1008) that accepts and boosts the audio input signal for output to both the various peripheral ports (i.e., the internal speaker, an external tape recorder, etc.) and the switching circuitry. The components of the audio amplifier are located inside the dotted line in Figure 3. The second component is the audio integrator circuitry which detects suprathreshold signals and generates a response. Varying levels of input sensitivity and response delay times are achieved by using variable strength resistors (i.e., potentiometers). The first resistor impedes the flow of current from the audio amplifier into the integrator circuitry. Higher levels of resistance to the incoming signal require stronger input signals to generate current flow downstream from the resistor. Once current flow below this first resistor is achieved, charge is held in a capacitor and allowed to dissipate at a rate controlled by the second resistor. High resistance levels at this second bottleneck produce longer decay periods for the capacitor, which results in sustained periods of current flow following the detection of a suprathreshold input signal. This current flow generates a response signal by closing a relay and completing a circuit along an output line with the recording device (the second of the two response methods described earlier). This completed circuit allows current to flow along the output line and into the input port of the recording device, thus mimicking the active generation of a response pulse. Circuit closure, and hence response signal production, is maintained for as long as there is sufficient charge held in the capacitor.

A number of factors influence the basic operational characteristics of the switch. Of these, the most important is the operational response time of the relay used to close the output circuit and produce a response signal. Our switch employs a 5-V mechanical relay (Radio Shack No. 275-243) with a 5-msec operation time and a 3-msec release. This means that there is a systematic 5-msec delay between the detection of a suprathreshold input signal and the generation of a response, as well as a $3-\mathrm{msec}$ delay in discontinuing that response once the input signal has ceased. For some users, the magnitude of these delays may prove unacceptable. In these cases, the mechanical relay may be replaced with a Reed digital relay. These relays have an operating time of $0.5 \mathrm{msec}$ with a 0.05 msec release and usually cost only $\$ 1$ to $\$ 2$ more than a mechanical relay. Mercury relays may also be substituted in lieu of a Reed relay, but the operating characteristics of these relays are often comparable to those of a Reed and they tend to be much more expensive.

A second factor influencing the overall operating characteristics of our switch involves the functional separation of the audio amplifier and the integrator circuitry. This separation has two important implications for the behavior of the switch. First, although input to the integrator is typically obtained from the built-in audio amplifier, inputs from an external amplifier can also be used. This feature allows an experimenter to boost, attenuate, or otherwise shape an incoming audio signal by using ex- ternal audio devices prior to its processing by the integration unit. The ability to use external amplifiers also allows the sensitivity range of the switch to be increased, since amplifiers that respond to a range of signals broader than the range to which the built-in amplifier responds can easily be used. Second, since the threshold sensitivity of the integrator component is dependent on the strength of the audio input signal, the threshold signal level above which the switch will respond is effectively set by a combination of both the volume level of the amplifier unit (either internal or external) and the sensitivity level of the integrator. Although this arrangement can occasionally prove awkward (e.g., every time the audio volume is decreased, the sensitivity level must be increased to offset the decrease in signal strength), it allows an experimenter to adjust the level of output to the peripheral devices (see Figure 2) independently of the output from the switch.

\section{Total Cost and Assembly Time}

The most expensive components of the unit consist of the audio amplifier $(\$ 11.95)$ and the steel utility box (\$6.79) in which the components are housed. The total cost for all parts was $\$ 35.65$ (not including tax), which is more than a 20 -fold reduction in price from that of the Gerbrands G1341-T (\$730). All parts were obtained from a standard Radio Shack mail-order catalogue. While the prices for individual components may vary somewhat, Radio Shack generally guarantees to maintain in stock all items listed in their catalogue or to replace them with some functional equivalent. The total time needed to assemble the unit was approximately $4.5 \mathrm{~h}$, including the usual time lost due to interruptions and errors in the assembly process. However, the actual time needed to assemble the unit will probably vary as a function of the level of expertise of the assembler. Users less experienced in assembling electronic components should plan on spending $10-20 \mathrm{~h}$ to assemble the switch.

\section{Reliability}

Data collected with the aid of this switch have been reported in several refereed journals. In one of these studies (Johnston et al., 1991, Experiment 7), some 8,448 latency measurements were made across 48 subjects. Average response latencies for each of the conditions of this study were comparable with those collected with the use of a Scientific Prototype Model 761-G when both switches were equated for frequency sensitivity and response delay time. Several of the these units are now employed in various labs at the University of Utah. Some of these switches are now several years old, and despite their regular use, they continue to function well. Owing to both the small number and general reliability of these switches, accurate estimates of breakdown and failure rates are not available. In our experience, most breakdowns stem from errors in the assembly process that place undo strain on one or more of the components. Other than failures in electronic components stemming from either current spikes or poor operating conditions, we expect that break- 
downs would be most likely to occur in the various mechanical components of the switch, such as the on/off and volume switches or the mechanical relay.

\section{CONCLUSION}

In this paper, we have described a sound-activated switch suitable for experiments utilizing vocal response times or other tasks involving the accurate detection of auditory onsets. This switch is comparable to a Gerbrands Model G1341-T in terms of its controls for sensitivity range and response signal characteristics, although the dynamic range for these variables is somewhat less. As a result, although our switch is adequate for detecting vocal response times in humans, it is probably inadequate for audio detection in species with vocalization ranges substantially higher or lower than that of humans (e.g., songbirds or bats). Although the sensitivity range could easily be extended by using an external amplifier with higher sensitivity characteristics than those of the built-in amplifier, this modification could substantially increase the cost of the unit. However, our switch does incorporate several additional features not provided on commercial switches, such as a built-in speaker and ports to facilitate the recording of the input signal. More importantly, the accuracy and durability of our switch appears to be comparable to that of commercially produced models, and it can be constructed for a fraction of the cost. As we mentioned in the introduction, vocal response times are used as indices for a variety of perceptual and memorial processes. Various computerized experimental generation systems capable of exploiting the capabilities of soundactivated switches are now available (e.g., PsyExpersee Hawley, 1991; MEL-see Schneider, 1988). When used in conjunction with these systems, the switch described in this paper can aid significantly in the timely and cost-effective design and conduct of experiments in which vocal response latencies are the primary dependent variable.

\section{REFERENCES}

Borden, G. J., \& HARRIS, K. S. (1980). Speech science primer. Baltimore: Williams \& Wilkins.

Brown, A. S., Neblett, D. R., Jones, T. C., \& Mitchell, D. B. (1991). Transfer of processing in repetition priming: Some inappropriate findings. Journal of Experimental Psychology: Learning, Memory, \& Cognition, 17, 514-525.
DARK, V. J. (1988). Semantic priming, prime reportability, and retroactive priming are interdependent. Memory \& Cognition, 16, 299-308.

Glaser, W. R., DUNGellhoff, F. (1984). The time-course of pictureword interference. Journal of Experimental Psychology: Human Perception \& Performance, 10, 640-654.

GlaSER, M. O., GLASER, W. R. (1982). Time course analysis of the Stroop phenomenon. Journal of Experimental Psychology: Human Perception \& Performance, 8, 875-894.

HAWLEY, K. J. (1991). PsyExper: Another experimental generation system for the IBM PC. Behavior Research Methods, Instruments, \& Computers, 23, 155-159.

Johnston, W. A., Hawley, K. J., \& Elllott, J. M. G. (1991), Contribution of perceprual fluency to recognition judgements. Journal of Experimental Psychology: Learning, Memory, \& Cognition, 17, 210-223

Levelt, W. J. M., Schriefers, H., Vorberg, D., Meyer, A. S, Pechmann, T., \& Havinga, J. (1991). The time course of lexical access in speech production: A study of picture naming. Psychological Review, 98, 122-142.

SCHNEIDER, W. (1988). Micro Experimental Laboratory: An integrated system for IBM PC compatibles. Behavior Research Methods, Instruments, \& Computers, 20, 206-217.

\section{NOTES}

1. Prices for commercially available sound-activated switches tend to vary according to the manufacturer and the extent of control over input and output waveforms provided by the switch. The Gerbrands Model G1341-T was chosen for comparison because of its similarity with the present switch in its control over input sensitivity and response generation. Its price should not be interpreted as being representative either of switches produced by other manufacturers or of other Gerbrands products.

2. Control of the response signal is to some degree dependent on the particular method of response production employed by any one switch. For example, while switches that generate an active response pulse may provide controls for the general waveform as well as the attack and decay periods of that pulse, switches that simply close a relay are rarely able to manipulate any signal characteristics other than the period of time for which circuit closure is maintained (i.e., a delay interval). In this secondary case, the response waveform is generally controlled by the device producing the current flow along the line connecting the switch and the input port of the recording device

3. In experimental attempts to detect vocal onsets for words embedded within a stream of continuous speech, it is typically necessary to adjust the response parameters of a switch so that a response is rapidly initiated following the detection of an input signal and yet decays completely during the acoustic interval between words. These task demands necessitate a much higher degree of control over the attack and decay of the response waveform than may be provided by more rudimentary switches and thus may influence the choice of a switch for a particular experiment.

(Manuscript received July 18, 1991; revision accepted for publication April 11, 1992.) 